\title{
Interface Analysis for Information interaction Between Smart Home Devices and Power Grid
}

\author{
Wenjing Li \\ State Grid Electric Power Research Institute \\ Beijing, China \\ e-mail: liwenjing@sgepri.sgcc.com.cn
}

\author{
Xinling Wu \\ State Grid Electric Power Research Institute \\ Beijing, China \\ e-mail: xinling-wu@sgepri.sgcc.com.cn
}

\begin{abstract}
Considering the programme of 95598 interactive services web site construction, models and application layer interface protocols for information interaction between smart home device and power grid has been proposed according to the main station existence of smart residential district. In this paper, smart grid services closely related with the power users were comprehensively reviewed, the requirements and contents of the interaction information among the entities are clarified and classified. Then, four kinds of interaction interface protocol in the application layer were proposed. The research of the information interaction interface in this paper is an initial attempt to standard the both-way information interaction, it also laid a theoretical basis to promote the development of energy efficiency management and enhancement of users' electricity experiences.
\end{abstract}

Keywords- Smart gri; smart home device; Information interaction model; Intelligent demand response

\section{INTRODUCTION}

With the rapid development of smart grid and the deepening of the AMI system, smart home and its energy efficiency management are increasingly becoming the research and application focuses of the power distribution and utilization communication network for smart grid.

The U.S., Europe, Japan, Korea and other countries have carried out a series of studies and explorations in intelligent community, buildings and residential district. They also obtained some experience and formed a related series of standard in the information interaction between Interaction between smart home device and power grid. There are several standardization organizations about smart home device communication in the foreign countries, mainly include DLNA, UOPF, ECHONET, ITOPHOME. Each standardization organization focuses on the specific business field. The standard of DLNA is mainly related to home entertainment and other business applications. ECHONET skills in home monitoring, but its criterions limit in the inner home network. The ECHONET criterions are mainly leaded by appliances and computer-related enterprises, power grid enterprises rarely participate, the information interaction between smart home device and power grid has not been researched comprehensively[1-4].

In recent years, intelligent community construction successfully combines the power-consuming information acquisition, distributed energy, smart electricity, smart home, power-consumption analysis and management together, which brings consumers the suitable, efficient, energy saving and environmental protection family life. With the rapid construction of the pilot project, the information between smart home device and power grid is becoming much more frequently. the consumers involved and interaction information are massively increasing. In this condition, the research of information interaction mode is critical. Our proposed scheme can efficiently ensure the service performance, enhance the information interaction efficiency and has great significance to guarantee the reliable operation and the security of power grid.

This paper mainly focused on the both-way data mode, content and application layer interface protocol of information interaction. The information interaction unification between smart home device and power grid in smart grid infrastructure has been attempted initially. The results in this paper can impel the construction of smart electricity service system, enhance the user QoS and comprehensively intelligentize the demand response to a certain extent.

\section{INFORMATION EXCHANGE MODELS AND PROTOCOL HIERARCHY}

\subsection{Information exchange models}

The Information exchange reference model between the smart home equipment and power grid is shown in Fig. 1. The information exchange includes two modes.

The main station of the intelligent community locates inner the community. Its construction need to make overall plans and coordinate with the data acquisition system, operating system of smart charging and exchanging service network for electric vehicles, data platform of energy efficiency management and 95598 interactive services website. The main station play an key role to connect the smart home device and power grid as the efficiency intermediary. The main station includes both-way interaction module, energy efficiency management module, smart home module and distribution access management module, which can be chosen and extend according to the desired function.

Both-way interaction module connects to 95598 website as an interface module. This module will realize the electricity data call from 95598 website to smart home device and the sending of appliance control command. energy efficiency management module will analyze the electricity information of smart home devices in order to form initial electricity strategy, then upload the processed information to energy efficiency management data platform 
in external information network. At last, the reasonable electricity-consuming suggesting will be given out to customers by coordinating. Smart home module can interpret commands and covert protocols for controlling signals issued by power grid. It can also send down the converted commands to smart home device and realize the smart controlling. distribution access management module is usually used to manage the related information of distributed power in users' side.

95598 websites are planed to two-level deploy and will cover large areas and many users. Along with the construction of smart grid, the number of users covered by 95598 website will continuously and obviously enhanced. If all the related information of smart home devices are processed by 95598 websites directly, the burden of 95598 websites will be too heavy and the efficiency running of power grid cannot be guaranteed.

The main station of intelligent district ions can worked as new part of grid and process the data of users and power grid initially. It can not only completely collects the information in user side but also efficiently mitigate pressure of the 95598 website. In addition, the main station can realize the user information management in detail by providing the community security information and other to consumers. In short, the main station is the essential entity to achieve the data exchange between smart home devices and power grid when there are a large number of users. If the user scale is small or with the construction of the operating platform, the main station can be regionally optional.

1) Mode 1

The smart home gateway connects with the 95598 website through the main station of intelligence community. The information of smart home devices is stored in main station and can be invoked by 95598 websites together with the public information of the intelligent community. The 95598 website interact with the gateway and control the running status of smart home devices through the main station.

2) Mode 2

The 95598 websites interacts with smart home gateway directly in order to exchange information with smart home devices and control the status.

2.2 Protocol hierarchy

The protocol hierarchy is shown as Fig. 2.

The physical layer can use wired or wireless way. IEEE802.11 、 IEEE 802.15.4 should be followed if use wireless way. Part of micro-power wireless and Bluetooth technologies should be convert protocols for do not supporting IP protocol. When use wired style, IEEE 802.3/IEEE 802.3ah/ IEEE P1901/ITU-T G.hn are preferred.

The network layer should support the IP protocol. The transport layer follows TCP/UDP protocol.

HTTP and SOAP are preferred in the application layer. The Web Service interface and its content should coincide with SG-CIM.

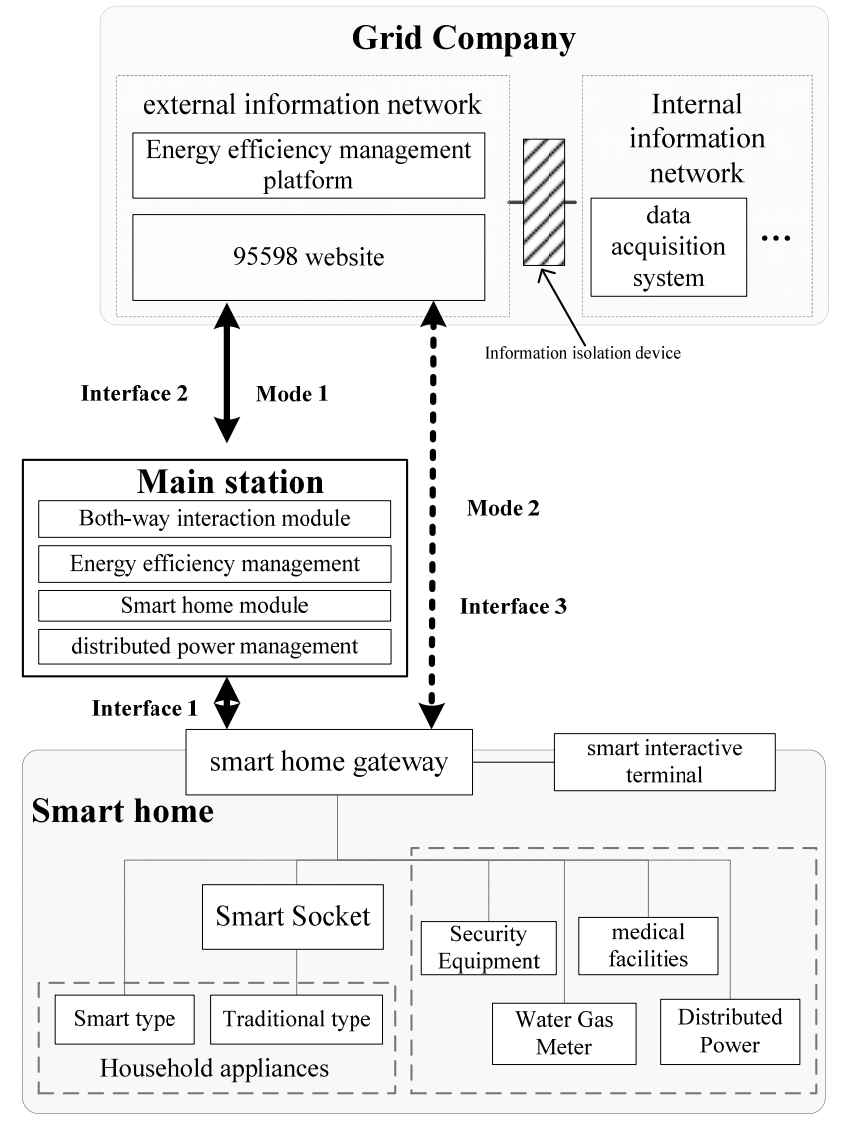

Figure 1. The Information exchange reference model.

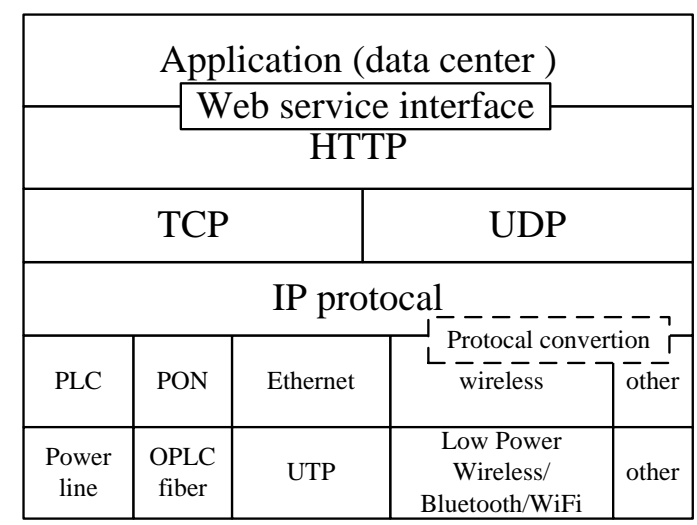

Figure 2. The protocol hierarchy

\section{INFORMATION EXCHANGE INTERFACE PROTOCOL IN THE APPLICATION LAYER}

The interface protocols of the information interaction between the smart home gateway and power grid include data Obtaining, data transmission, event and register protocols. The connection between entities adopted the Web Service style based on HTTP criterion, the interface message uses the SOAP protocol. 


\subsection{Data Obtaining protocol}

The data obtaining is a protocol for data retrieval from a remote component. It can be used to realize data obtaining between smart home gateway and power grid. The entity required data is denoted as the requester, the entity provide data is called provider. Fig. 3 provides a diagram of the interaction.

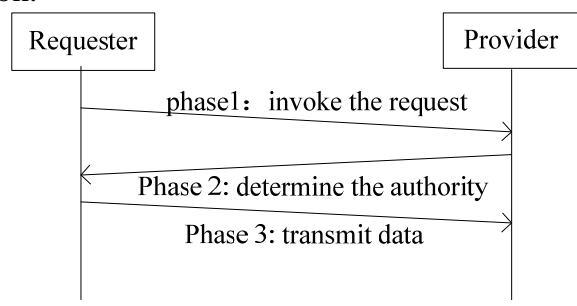

Figure 3. The diagram of the interaction

Phase 1: Requester invokes the query method of its Provider. The Requester shall send query information including required content, effective time and the size of acceptable dataset. If the last two parameters have not been fixed, the system default settings will be used.

Phase 2: Identity and authority of requester will be determined by the provider at first. If the request is valid the Provider returns a subset of the whole dataset. If the size of the returning dataset is exceeding the acceptable data size of requester, the provider returns only a subset of the whole data set. For succeeding data retrieval, the provider also returns the associated data step-by-step.

Phase 3: Requester estimates according to the received data. If all data has been accepted, then this process completes. If only part of data has been received, the Requester invokes the query method at the Provider again (with the given cursor), and go to Phase 2 .

\subsection{Data transmission protocol}

The data transmission is a protocol for data transfer to a remote component. We denote the component that submits data to the remote component by Requester, and the component that receives the data by Target. Fig. 4 provides a diagram of the interaction.

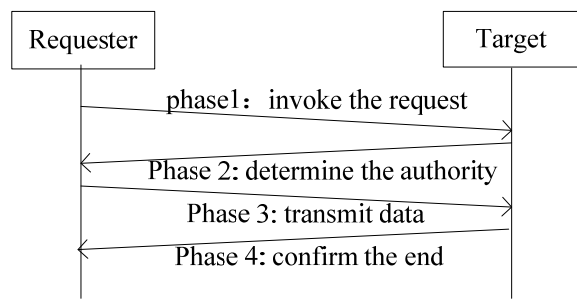

Figure 4. The diagram of the interaction

Phase.1:.Requester invokes the transmit request to its Target. The Requester shall send requester information including entity identification, effective time and the size of acceptable dataset. If the last two parameters have not been fixed, the system default settings will be used.

Phase 2: Identity and authority of requester will be determined by the target at first. If the request is valid the Target returns confirmation message.
Phase.3:.Requster transmits data to target including its content and finished flag.

Phase 4: Target estimates according to the received data. If all data has been accepted, then this process completes, else returns the error message.

\subsection{Event protocol}

The Event is a protocol for instruction exchanging between smart home gateway and power grid. We denote the component that invokes the event by "Event Requester", the component that receives and implement the event by "executor", and the component that sends down the event request by "issued entity". Fig. 5 provides a diagram of the interaction.

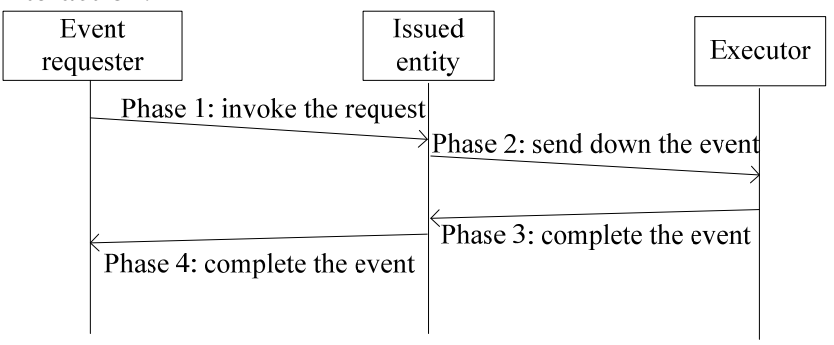

Figure 5. The diagram of the interaction

Phase 1: Requester invokes the event issuing request to its issued entity. The Requester shall send requester information including identifications of the event requester and executor, effective time and event contents.

Phase 2: Identity and authority of event requester will be determined by the issued entity at first. If the request is valid the issued entity sends the event down to the executor.

Phase 3: Identity and authority of event requester will be determined by the Executor at first. If the request is valid the Executor implements the event content and return finished message to the issued entity.

Phase 4: The issued entity return the finished message to the event requestor.

\section{4 register protocol}

The register is a protocol to authenticate the legal status and authority when information interactive. this protocol is the basic one that always occurred before all other three protocols in order to ensure the reliability and validity. We denote the component that submits registration request to its Registry by "Registrant". and the component that authenticates the data by "Registry". Fig. 6 provides a diagram of the interaction.

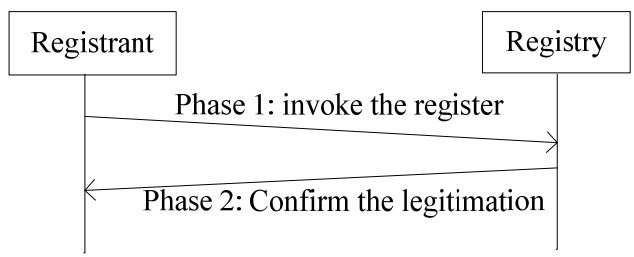

Figure 6. The diagram of the interaction 
Phase 1: Registrant invokes the registration method of the Registry with the component information (e.g., the name, the access URI of the component, and protocol types this component supports) and its role information (e.g., what Point IDs this component manages).

Phase 2: The Registry replies whether it was successful or not. If it was not, it shall return error message to the Registrant.

\section{CONCLUSION}

In this paper, considering the program of 95598 interactive service websites and the deployment of the smart electricity system, two interactive modes and four interface protocols in application layer have been proposed according whether the main station exists or not. With comprehensively reviewing the smart electricity businesses related with power consumers, this paper defined the requirement and interaction content and four kinds of Web Service interface protocols in the application layer. The results in this paper can impel the construction of smart electricity service system, enhance the user QoS and comprehensively intelligentize the demand response to a certain extent.

\section{REFERENCES}

[1] Young-Sung Son, Pulkkinen, T., Kyeong-Deok Moon, Chaekyu Kim, 2010, "Home energy management system based on power line communication", IEEE Transactions on Consumer Electronics, vol.56, 1380-1386.

[2] Dae-Man Han; Jae-Hyun Lim, 2010, "Smart home energy management system using IEEE 802.15.4 and zigbee", IEEE Transactions on Consumer Electronics, vol.56, 1403-1410.

[3] Yong Zhao; Wanxing Sheng; Junping Sun; Weijun Shi, 2011, "Research and thinking of friendly smart home energy system based on smart power", International Conference on Electrical and Control Engineering (ICECE).

[4] Liu, C.W.; Luo, C.C.; Lin, P.Y.; Lu, G.C.; Wu, W.C.,"Develop a power quality measurement system integrated with HAN home energy management system", 2011 4th International Conference on Electric Utility Deregulation and Restructuring and Power Technologies (DRPT), 2011, 1506-1510 